\title{
Short range nucleon correlations studied with electron and photon probes
}

\author{
I. J. Douglas MacGregor ${ }^{\star}$ \\ The University of Glasgow, Glasgow, UK \\ $\star$ douglas.macgregor@glasgow.ac.uk \\ Proceedings for the 24th edition of European Few Body Conference, \\ Surrey, UK, 2-6 September 2019 \\ doi:10.21468/SciPostPhysProc.3
}

\begin{abstract}
This paper reviews experimental research into two- and three-body nucleon-nucleon interactions, carried out by the University of Glasgow Nuclear Physics research group. A key aim of these studies has been to elucidate the role of short-range nucleon-nucleon correlations (SRC). Studies of photon-induced two- and three-nucleon emission reactions from a range of light nuclei, carried out at the $840 \mathrm{MeV}$ Mainz electron microtron MAMI-B, have provided detailed information on the contributing mechanisms. More recent electron scattering studies at the $6.0 \mathrm{GeV}$ Jefferson Laboratory have probed SRC at high values of $Q^{2}$ and $x_{B}$ and suggested a connection between SRC and the EMC effect.
\end{abstract}

(c) (1) Copyright I. J. D. MacGregor.

This work is licensed under the Creative Commons

Attribution 4.0 International License.

Published by the SciPost Foundation.
Received 30-10-2019

Accepted 20-12-2019

Published 24-02-2020

doi:10.21468/SciPostPhysProc.3.010

\section{Contents}

1 Introduction $\quad 2$

$2(\gamma, \mathrm{pN})$ Measurements at Mainz $\quad 2$

$3 \quad\left(e, e^{\prime}\right)$ Measurements at Jefferson Lab $\quad 8$

4 Photon-Induced 3-Nucleon Knockout Experiments $\quad 11$

5 Summary and Conclusions $\quad 12$

$\begin{array}{ll}\text { References } & 14\end{array}$ 


\section{Introduction}

In a 1993 review [1] of quasi-elastic (e,e'p) experiments carried out over the preceding few years, on a range of nuclear targets from ${ }^{7} \mathrm{Li}$ to ${ }^{208} \mathrm{~Pb}$, Lapikás noted a large systematic reduction in the spectroscopic strength of valence orbitals, compared to nuclear mean-field models. This reduction was in the range $30 \%-40 \%$, varying slightly between different nuclei. In contrast, the measured occupancies of states just above the Fermi surface in the same nuclei were observed to be greater than zero, with values between $5 \%$ and $13 \%$. These observations were consistent with a shifting of strength from valence to higher-energy orbitals, due to short-range and tensor correlations, not included in the mean-field nuclear models.

Prior to this, in the mid-1980s, the Glasgow nuclear physics research group had started a systematic programme of photon- and electron-induced two-nucleon emission experiments at the Mainz electron microtron facility MAMI, to investigate the nature of possible correlations between pairs of nucleons. Most of this work used the tagged photon technique, exploiting bespoke tagged photon spectrometers designed and constructed by the Glasgow group [2,3]. This work studied the $(\gamma, \mathrm{pn}),(\gamma, \mathrm{pp})$ and $\left(\mathrm{e}, \mathrm{e}^{\prime} \mathrm{pn}\right)$ reactions in a range of light nuclei: ${ }^{3} \mathrm{He}[4,5]$, ${ }^{4} \mathrm{He}$ [6], ${ }^{6} \mathrm{Li}[7],{ }^{12} \mathrm{C}$ [8-18] and ${ }^{16} \mathrm{O}$ [19-21]. The most extensive investigations were carried out on ${ }^{12} \mathrm{C}$, but the results on other light nuclei provided a similar and consistent interpretation.

The real photon measurements studied several different aspects of two-nucleon emission reactions including their photon energy dependence, missing energy dependence, recoil momentum dependence and angular dependence, as well as their isospin dependence and their dependence on photon polarisation. Collaborations were formed with theoretical groups working in Valencia, Pavia and Gent in order to provide a detailed interpretation of the data obtained. This work is summarised in Sec.2.

More recent work at the Jefferson Lab $6.0 \mathrm{GeV}$ electron accelerator has focussed on SRC at higher energies, in a range of nuclei up to ${ }^{208} \mathrm{~Pb}$. This work used both inclusive $\left(\mathrm{e}, \mathrm{e}^{\prime}\right)$ [22] and exclusive (e, $\left.\mathrm{e}^{\prime} \mathrm{pn}\right)$ [23] experiments at high $\mathrm{x}_{B}$ and high $\mathrm{Q}^{2}$ to study nucleon correlations at high relative nucleon momenta. This work obtained the probabilities of virtual photons interacting with correlated nucleon pairs, or correlated triples, as $x_{B}$ is increased.

Semi-inclusive $\left(e, e^{\prime} p\right)$ and $\left(e, e^{\prime} n\right)$ reactions $[24,25]$ have shown that the strong isospin dependence of two-nucleon correlations, noted in the earlier Mainz work, namely the strong suppression of interactions with proton-proton pairs, extends to high energies, even though the detailed mechanisms are different. Moreover this semi-inclusive electron scattering work has identified a strong isospin dependence in the fraction of nucleons carrying high momentum which can be understood as a direct consequence of the isospin dependence of SRC.

Very recent work [26] has suggested a connection between SRC and the EMC effect, in which the structure function $F_{2}\left(\mathrm{x}_{B}, \mathrm{Q}^{2}\right)$ observed in Deep Inelastic Scattering (DIS) on nucleons bound in heavy nuclei is suppressed, compared to the same reaction on nucleons in light nuclei. The results of these studies are discussed in Sec.3.

The contribution of three-body interactions to two- and three-nucleon emission reactions was also investigated with real photons at Mainz. This work included studies of the ${ }^{12} \mathrm{C}(\gamma, \mathrm{ppn})$, ${ }^{12} \mathrm{C}(\gamma, \mathrm{pnn})$ and ${ }^{12} \mathrm{C}(\gamma, \mathrm{pd})$ reactions [27-30]. See Sec.4.

\section{$2(\gamma, \mathrm{pN})$ Measurements at Mainz}

Starting in the mid-1980s, the Glasgow nuclear physics research group carried out a series of $(\gamma, \mathrm{pN})$ experiments at the Mainz electron microtron MAMI. The earliest experiments were performed at the $180 \mathrm{MeV}$ accelerator (MAMI-A). Higher energies became available in 1990 when the Mainz accelerator was upgraded to $840 \mathrm{MeV}$ (MAMI-B). Fig. 1 shows a typical 


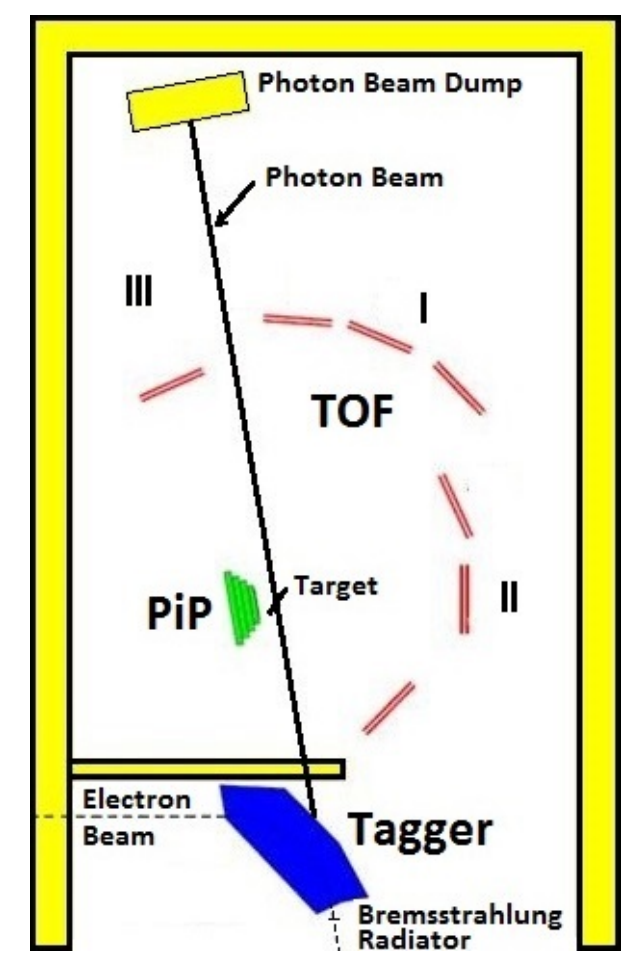

Figure 1: Experimental detector layout for $(\gamma, \mathrm{pN})$ experiments carried out at the Mainz $840 \mathrm{MeV}$ electron microtron MAMI-B. Tagged photons are produced in a Bremsstrahlung Radiator, placed in the electron beam before the Glasgow tagged photon spectrometer (Tagger) [3]. For linearly polarised photons an aligned diamond radiator is used [32]. The scattered bremsstrahlung electron energies $E_{e}^{\prime}$ are measured in the Tagger. Knowing the electron beam energy $E_{e}$, the energy of each photon can then be deduced: $E_{\gamma}=E_{e}-E_{e}^{\prime}$. Protons ejected from the Target are detected in the Pion and Proton charged particle hodoscope PiP [33], which acts as a trigger detector. Correlated neutrons and protons are detected using a series of TOF time-of-flight scintillator detector arrays [34], set at a range of polar angles I, II and III covering diffferent kinematic settings.

experimental detector layout from one of the experiments carried out at MAMI-B.

Fig. 2 shows missing energy $\left(E_{m}\right)$ spectra for ${ }^{12} \mathrm{C}(\gamma, \mathrm{pn})$ and $(\gamma, \mathrm{pp})$ reactions, measured by Lamparter et al. [12], for $\mathrm{E}_{\gamma}$ between 114 and $600 \mathrm{MeV}$. The $(\gamma, \mathrm{pn})$ data show a sharp peak at low missing energies $\left(E_{m}\right)$, followed by a broader distribution at higher values of $E_{m}$. The peak is most prominent at low $\mathrm{E}_{\gamma}$, but remains discernible at higher $\mathrm{E}_{\gamma}$ values. Comparisons are made with predictions from the Valencia Model (VM) [31], averaged over the detector acceptances.

This model uses a simple Fermi gas approach with a local density approximation. It calculates all of the the expected contributing mechanisms and treats FSI of the emitted particles semi-classically. However, as it does not contain any detailed nuclear structure information, it is only able to reproduce the general features of the reaction process.

The VM reproduces both the strength and shape of the $(\gamma, \mathrm{pn}) \mathrm{E}_{m}$ spectra reasonably well. The good quality of the comparison indicates that direct absorption on proton-neutron pairs occurs at low $\mathrm{E}_{m}$, with final state interactions (FSI) extending strength to higher values of $\mathrm{E}_{m}$. Photon absorption on three-nucleon clusers (with FSI) and quasifree pion emission, with pion scattering or reabsorption, all produce strength at higher $\mathrm{E}_{m}$.

The $(\gamma, \mathrm{pp})$ strength at low $\mathrm{E}_{m}$ is a factor of $\sim 40$ weaker than the $(\gamma, \mathrm{pn})$ channel at the 


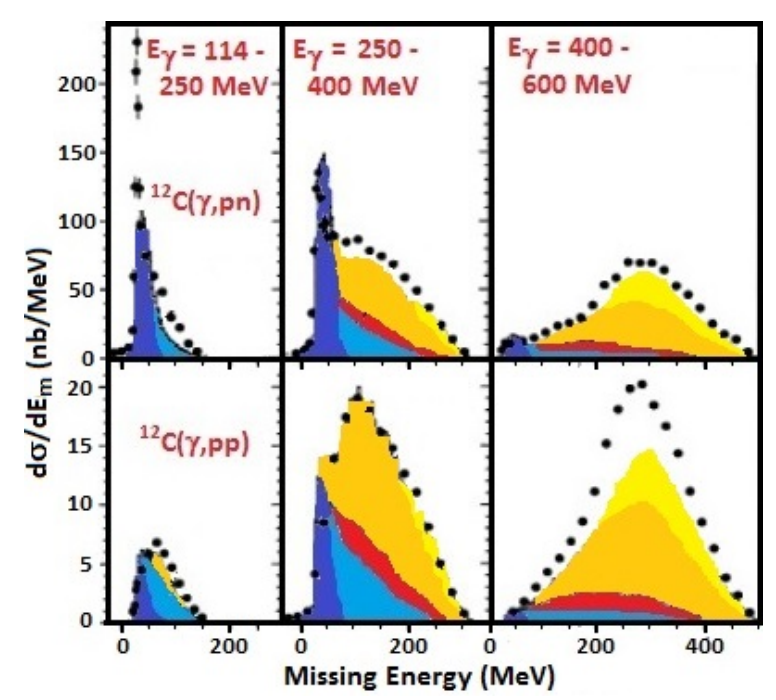

Figure 2: ${ }^{12} \mathrm{C}(\gamma, \mathrm{pn})$ and $(\gamma, \mathrm{pp}) \mathrm{E}_{m}$ spectra [12], for three photon energy ranges up to $600 \mathrm{MeV}$, compared to predictions from the VM [31]. Dark blue indicates photon absorption on two nucleons; light blue is photon absorption on two nucleons with FSI; red indicates photon absorption on three nucleons, with or without FSI; orange represents quasi-free pion production where the pion is subsequently reabsorbed and yellow corresponds to quasi-free pion production where the pion rescatters. The $(\gamma, \mathrm{pp})$ calculations have been reduced in magnitude by a factor of 3.5.

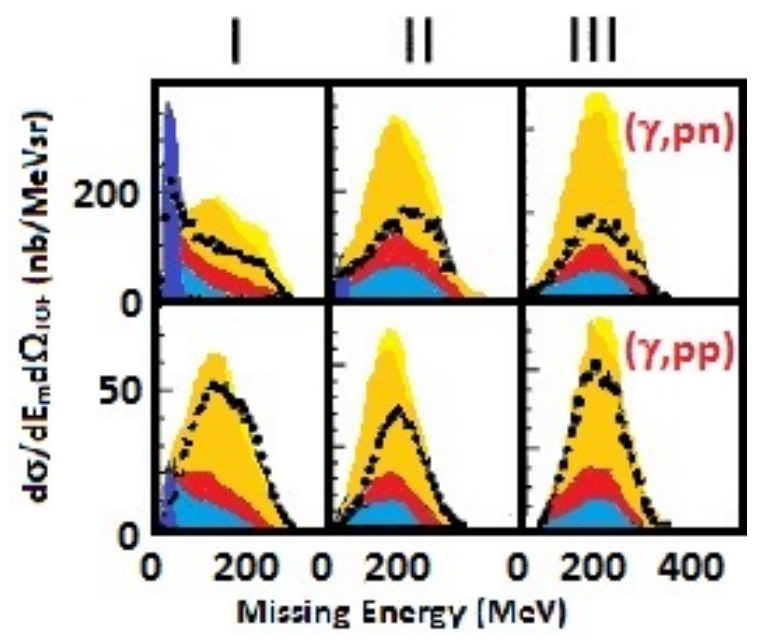

Figure 3: ${ }^{12} \mathrm{C}(\gamma, \mathrm{pn})$ and $(\gamma, \mathrm{pp})$ differential cross sections as a function of $\mathrm{E}_{m}$ [16], for $\mathrm{E}_{\gamma}=300-400 \mathrm{MeV}$. Region I covers quasi-deuteron kinematics (back-to-back nucleon emission in the $\mathrm{CM}$ frame of the photon and a stationary nucleon pair); Region II corresponds to non-quasideuteron kinematics, with wider particle opening angles; Region III is extreme kinematics in which both emitted nucleons are on the same side of the photon beam. The experimental spectra are compared to predictions of the VM. The colours have the same interpretation as in 2 .

lowest $E_{\gamma}$, although this factor reduces rapidly as $E_{\gamma}$ increases. It has no discernible peak at low $\mathrm{E}_{m}$, but rather has a broader distribution. The VM calculations do not correctly predict the $(\gamma, \mathrm{pp})$ strength and have been reduced by a factor of 3.5. They do, however, give a reasonable account of the shape of the $\mathrm{E}_{m}$ spectra. It is clear that direct $2 \mathrm{~N}$ absorption plays a smaller role in the $(\gamma, \mathrm{pp})$ channel, compared to the other contributing mechanisms. The relative 
contribution of $3 \mathrm{~N}+\mathrm{FSI}$ processes is similar in both channels.

To investigate how the reaction cross section, and its contributing mechanisms, varied with the opening angle between the proton detected in PiP and the second nucleon, Watts et al. [16] studied three different kinematic settings. Direct absorption on two-nucleon pairs was only appreciable in Region I (quasi-deuteron kinematics), see Fig. 3. The non-quasideuteron and extreme settings (Regions II and III) emphasised processes involing FSI, 3N-absorption or quasi-free pion production. As a method of selecting $3 \mathrm{~N}$ processes, these kinematics enhanced the $3 \mathrm{~N}$ contribution, but not sufficiently for it to be considered a dominant process.

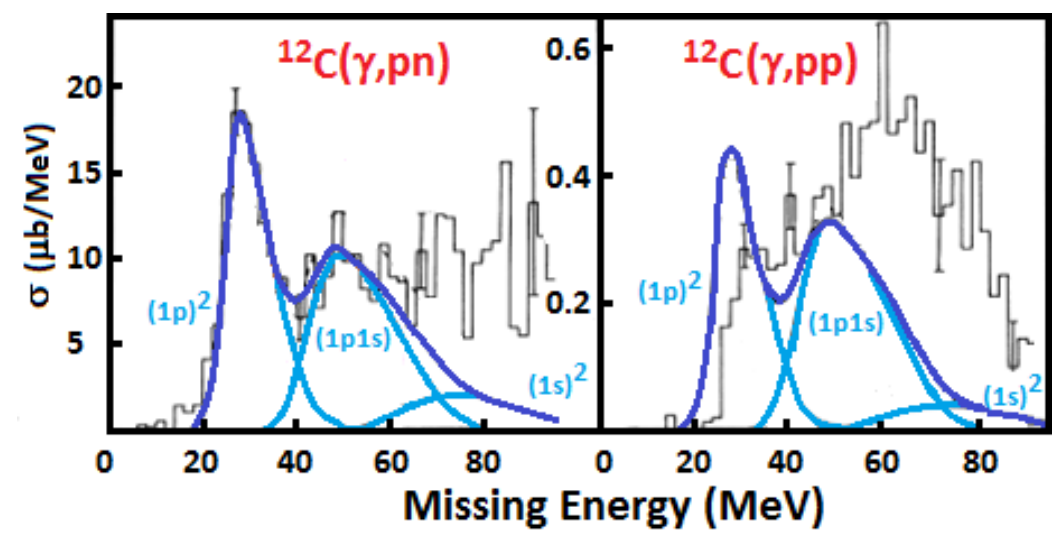

Figure 4: ${ }^{12} \mathrm{C}(\gamma, \mathrm{pn})$ and ${ }^{12} \mathrm{C}(\gamma, \mathrm{pp}) \mathrm{E}_{m}$ spectra [9], for $\mathrm{E}_{\gamma}=145-157 \mathrm{MeV}$. The data are compared to spectral shapes obtained by folding together two $\left(e, e^{\prime} \mathrm{p}\right)$ spectra, with the $7 \mathrm{MeV}$ experimental energy resolution. The strength of emission from (1p) ${ }^{2}$, $(1 \mathrm{p} 1 \mathrm{~s})$ and $(1 \mathrm{~s})^{2}$ orbitals is detemined by the number of available nucleon pairs.

McGeorge et al. [9] compared the shape of the $\mathrm{E}_{m}$ spectra for ${ }^{12} \mathrm{C}(\gamma, \mathrm{pn})$ and ${ }^{12} \mathrm{C}(\gamma, \mathrm{pn})$ with two $\left(e, e^{\prime} p\right)$ one nucleon emission spectral shapes, folded together with the known experimental resolution (see Fig. 4). The strength of emission from different orbitals was detemined by the number of available nucleon pairs. This worked well for low $\mathrm{E}_{m}$ for both reaction channels, giving a further indication that cuts on $\mathrm{E}_{m}<40 \mathrm{MeV}$ could be used to select direct absorption on nucleons in $(1 \mathrm{p})^{2}$ orbitals and a cut from $40-70 \mathrm{MeV}$ predominantly selected absorption on (1p1s) pairs. Other mechanisms are needed to explain the observed strength at higher $\mathrm{E}_{m}$.

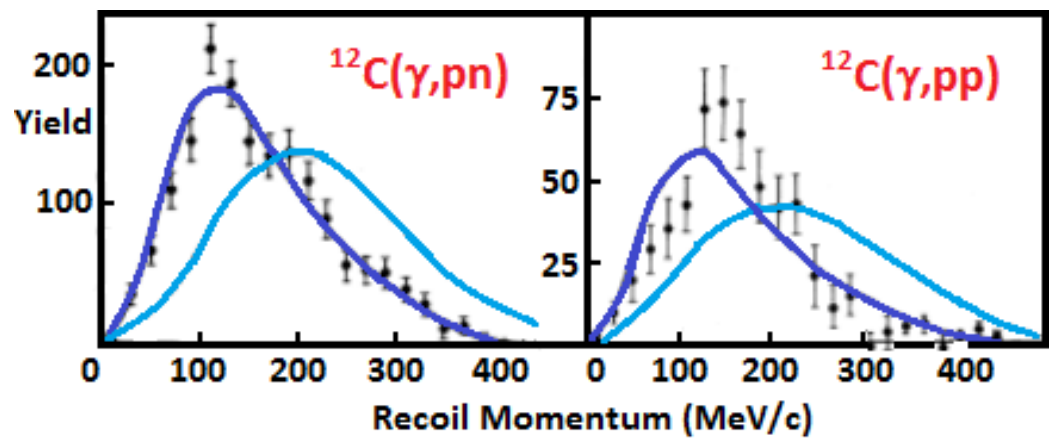

Figure 5: ${ }^{12} \mathrm{C}(\gamma, \mathrm{pn})$ and ${ }^{12} \mathrm{C}(\gamma, \mathrm{pp})$ recoil momentum spectra [9], for $\mathrm{E}_{\gamma}=145-157$ $\mathrm{MeV}$ and $\mathrm{E}_{m}<40 \mathrm{MeV}$. The data are compared to Monte Carlo calculations of twonucleon photon absorption (dark blue) and Phase Space (light blue). See text for details.

Recoil momentum spectra provide a good test as to whether any residual FSI affect the selected events. Fig. 5 shows the recoil distributions for ${ }^{12} \mathrm{C}(\gamma, \mathrm{pn})$ and $(\gamma, \mathrm{pp})$ events for $\mathrm{E}_{m}<40$ $\mathrm{MeV}$. The data are compared with two Monte Carlo models. The $2 \mathrm{~N}$ absorption model folds 
together two Harmonic Ooscillator momentum wavefunctions, filtered by the detector acceptance. Also shown is a phase space distribution. Neither reaction agrees with the phase space distribution, indicating the two emitted nucleons are strongly correlated. The $(\gamma, \mathrm{pn})$ data fits the $2 \mathrm{~N}$ absorption model extremely well, while the fit to the $(\gamma, \mathrm{pp})$ channel is reasonable.

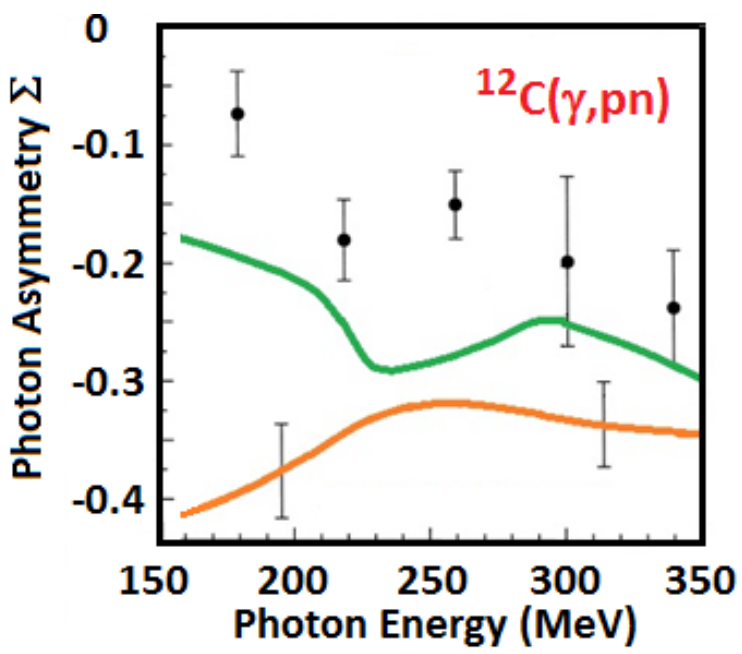

Figure 6: ${ }^{12} \mathrm{C}(\gamma, \mathrm{pn})$ photon asymmetry $\Sigma$ data [15], for $\mathrm{E}_{m}<40 \mathrm{MeV}$. The photon asymmetry is defined by $\Sigma=\left(\sigma_{\|}-\sigma_{\perp}\right) /\left(\sigma_{\|}+\sigma_{\perp}\right)$ where $\sigma_{\|}\left(\sigma_{\perp}\right)$ is the cross section parallel (perpendicular) to the plane of polarisation of the incident photon. The data are compared to an average asymmetry from previous $d(\gamma, p n)$ measurements $[35,36]$ (green line) and to calculations made using the Gent Model (orange line), described in the text.

The photon asymmetry $\Sigma$ is an observable sensitive to transverse components of the electromagnetic interaction, including spin variables and interference between contributing terms in the nuclear current. It is instructive to use $\Sigma$ to test some of the dynamical aspects of quasideuteron photon absorption. Fig. 6 shows $\Sigma$ for the ${ }^{12} \mathrm{C}(\gamma, \mathrm{pn})$ reaction for $\mathrm{E}_{\gamma}$ between 150 and $350 \mathrm{MeV}$, measured by Franczuk et al. [15]. The data show a moderate negative asymmetry with a slight hint of structure in the $\Delta$-resonance region. The data are compared to an average of previous $\mathrm{d}(\gamma, \mathrm{pn})$ data $[35,36]$, shown in green. The measured $\Sigma$-values are consistently smaller in magnitude than the corresponding deuterium values. This indicates that proton-neutron pair emission from light nuclei is a more complex process than a simple scaling of deuterium photodisintegration. The data are also compared with a microscopic distorted wave model developed by the Gent theory group, shown in orange. This model includes the $\pi$-seagull, $\pi$-in-flight and $\Delta$-excitation mechanisms, as well as SRC. Although including all of the expected ingredients, this model does not agree with the data, predicting a significantly larger asymmetry magnitude.

Measurements in the $(\gamma, \mathrm{pp})$ channel by Powrie et al. [17] show a very interesting feature: namely a strong negative peak at low $\mathrm{E}_{m}$. This is shown in Fig. 7 for $\mathrm{E}_{\gamma}=160-220 \mathrm{MeV}$. The magnitude of this peak is stronger than the measured asymmetry magnitude for ${ }^{12} \mathrm{C}(\gamma, \mathrm{pn})$. The shape and magnitude of the asymmetry indicates a distinct two-nucleon absorption mechanism for the $(\gamma, \mathrm{pp})$ channel at low $\mathrm{E}_{m}$.

The fact that the $(\gamma, \mathrm{pp})$ asymmetry has a larger magnitude than $(\gamma, \mathrm{pn})$ rules out $(\gamma, \mathrm{pp})$ being strongly fed by charge-exchange FSI from the stronger $(\gamma, \mathrm{pn})$ channel. It is also expected that any FSI will tend to dilute the photon asymmetry of the initial process. This is seen at higher $\mathrm{E}_{m}$ in Fig. 7 , where FSI effects are thought to dominate the $(\gamma, \mathrm{pp})$ channel, and the magnitude of $\Sigma$ is considerably lower.

Asymmetry measurements at photon energies up to $350 \mathrm{MeV}$ (not shown here), and in 


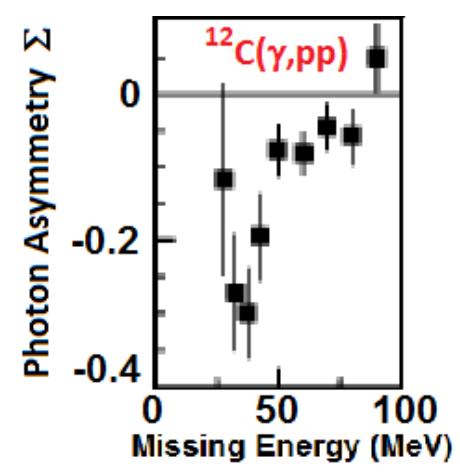

Figure 7: ${ }^{12} \mathrm{C}(\gamma, \mathrm{pp})$ photon asymmetry $\Sigma$ measurments [17] as a function of missing energy, for $\mathrm{E}_{\gamma}=160-220 \mathrm{MeV}$.

${ }^{16} \mathrm{O}$ [17], show that this strong negative structure at low missing energies persists at higher values of $\mathrm{E}_{\gamma}$ and does not depend on the particular target nucleus being studied.

MacGregor et al. [13] carried out further studies to investigate the $\mathrm{E}_{\gamma}$ dependence of the ${ }^{12} \mathrm{C}(\gamma, \mathrm{pn})$ and ${ }^{12} \mathrm{C}(\gamma, \mathrm{pp})$ reaction cross sections and their angular dependence. In these measurements both the PiP and ToF detectors were rotated round the target position, while maintaining the "back-to-back" quasi-deuteron kinematics.

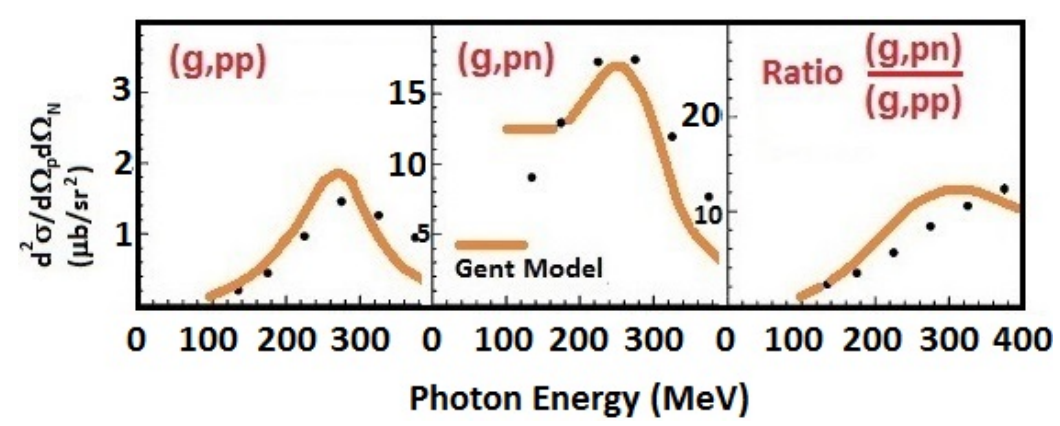

Figure 8: $\mathrm{E}_{\gamma}$ dependence of ${ }^{12} \mathrm{C}(\gamma, \mathrm{pp})$ and ${ }^{12} \mathrm{C}(\gamma, \mathrm{pn})$ double differential cross sections, and their ratio, for $\mathrm{E}_{\gamma}=100-400 \mathrm{MeV}$ and $\mathrm{E}_{m}<40 \mathrm{MeV}$ [13], compared to calculations using the Gent Model (see text).

Fig. 8 shows the energy dependence of the ${ }^{12} \mathrm{C}(\gamma, \mathrm{pp})$ and ${ }^{12} \mathrm{C}(\gamma, \mathrm{pn})$ double differential cross sections, and their ratio, for $E_{\gamma}=100-400 \mathrm{MeV}$ and $E_{m}<40 \mathrm{MeV}$ in quasi-deuteron kinematics [13]. Both reactions show a peak in the $\Delta$-resonance region, indicating that $\Delta$ currents make a strong contribution at these energies. The $(\gamma, \mathrm{pp})$ reaction is much weaker than the $(\gamma, \mathrm{pn})$ reaction. However, the $(\gamma, \mathrm{pn}) /(\gamma, \mathrm{pp})$ ratio increases steadily with $\mathrm{E}_{\gamma}$.

The data are compared to one-pion-exchange calculations made using the Gent Model [37]. These theoretical calculations use an unfactorised approach including a dynamic treatment of the $\Delta$ propagator. The Gent Model is able to reproduce the general features of energy dependence of the double differential cross sections. It also provides a reasonable account of both the strength and the shape of both reaction channels.

Fig. 9 shows the angular distribution of both reactions, measured for $\mathrm{E}_{m}<40 \mathrm{MeV}$ and $\mathrm{E}_{\gamma}=200-300 \mathrm{MeV}$ in quasi-deuteron kinematics. The data are again compared with predictions of the Gent Model [37]. The cross section is plotted as a function of the polar angle of the proton detected in PiP. The angular distributions of both channels are seen to be very different. The $(\gamma, \mathrm{pn})$ reaction peaks at central proton angles, whereas the $(\gamma, \mathrm{pp})$ channel has a clear dip at central laboratory angles. Strong differences between the angular distributions of both channels are also observed at lower and higher photon energies, not shown here [13]. 


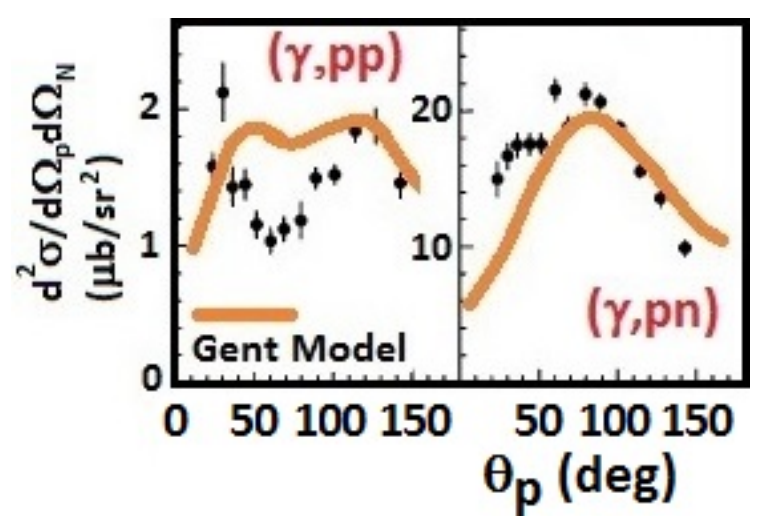

Figure 9: Angular distributions of ${ }^{12} \mathrm{C}(\gamma, \mathrm{pp})$ and ${ }^{12} \mathrm{C}(\gamma, \mathrm{pn})$ double differential cross sections, for $\mathrm{E}_{\gamma}=200-300 \mathrm{MeV}$ and $\mathrm{E}_{m}<40 \mathrm{MeV}$ in quasi-deuteron kinematics [13], compared to calculations using the Gent Model [37] (see text).

These differences are a clear indication of distinct reaction mechanisms in the two channels. In particular, the measured data discount the possibility that the weaker $(\gamma, \mathrm{pp})$ is simply fed by FSI from the stronger $(\gamma, \mathrm{pn})$ channel.

The Gent Model is able to reproduce the general strengths and some of the angular features of both angular distributions, but does not provide close agreement with the distributions in either channel. Further improvements in the theoretical treatments, including the inclusion of heavier mesons and improved treatments of SRC, are required before we can obtain a full understanding of the contributing reaction mechanisms, even where a clean sample of twonucleon knockout events, free of FSI, have been separated out.

\section{$3\left(e, e^{\prime}\right)$ Measurements at Jefferson Lab}

A more direct approach to study SRC is to use inclusive (e, $\left.\mathrm{e}^{\prime}\right)$ electron scattering at high values of $\mathrm{Q}^{2}$ (4-momentum transfer) and the Bjorken scaling variable $\mathrm{x}_{B}=Q^{2} / 2 M v$, where $v$ is the energy transfer from the electron to the target nucleon and $M$ is the nucleon mass. SRC between pairs of nucleons are characterised by both interacting nucleons having large (and opposite) momentum values. These high momentum components of the nucleon wavefunction can be probed by virtual photons with large values of both $\mathrm{Q}^{2}$ and $v$. Generally when a virtual photon inteacts with a SRC pair, knocking out one nucleon, the other nucleon will also be knocked out. This happens irrespective of whether or not the ejected nucleons are subsequently detected.

Egiyan et al. [22] looked for signatures of SRC in a range of nuclei $\left({ }^{4} \mathrm{He},{ }^{12} \mathrm{C}\right.$ and $\left.{ }^{56} \mathrm{Fe}\right)$ using exclusive $\left(\mathrm{e}, \mathrm{e}^{\prime}\right)$ reactions at $\mathrm{Q}^{2}>1.4(\mathrm{GeV} / \mathrm{c})^{2}$ and $1.0<\mathrm{x}_{B}<3.0$ at the CLAS facility at Jefferson Lab. As exclusive electron scattering cross sections drop rapidly with $\mathrm{x}_{B}$, the approach taken was to plot the ratio of scaled exclusive cross sections in each nucleus to that in ${ }^{3} \mathrm{He}$, which contains just three nucleons. The results are shown below in Fig. 10.

In general, cross sections at $\mathrm{x}_{B}>1$ require the involvement of more than one nucleon, and for $\mathrm{x}_{B}>2$ more than two nucleons are required. The results show a clear scaling of the cross section ratios in the regions, $1.6<\mathrm{x}_{B}<2.0$ and $2.3<\mathrm{x}_{B}<2.7$. This is taken as a clear indication of direct interaction with two-nucleon SRC pairs and three-nucleon SRC triples. Moreover the observation of similar scaling in different light, medium and heavy nuclei, strongly suggest that SRC are a feature of nucleon-nucleon interactions in all nuclei. The behaviour in the regions $1.0<\mathrm{x}_{B}<1.6$ and $2.0<\mathrm{x}_{B}<2.3$, where there is not a strict scaling, is likely to be due 


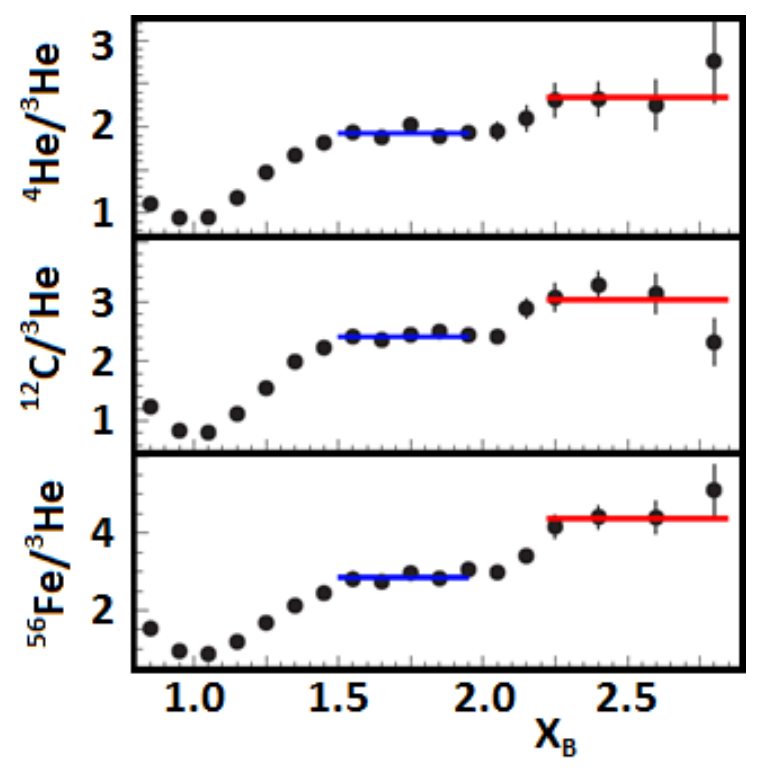

Figure 10: Ratios of exclusive (e,e $\left.\mathrm{e}^{\prime}\right)$ cross sections for ${ }^{4} \mathrm{He},{ }^{12} \mathrm{C}$ and ${ }^{56} \mathrm{Fe}$ to ${ }^{3} \mathrm{He}$, as a function of $\mathrm{x}_{B}$ for $\mathrm{Q}^{2}>1.4(\mathrm{GeV} / \mathrm{c})^{2}$ [22]. It is expected that interactions with SRC two-nucleon pairs will occur for $1<\mathrm{x}_{B}<2$ and with three-nucleon triples for $2<\mathrm{x}_{B}<3$ (see text). The blue and red lines show plateaux observed in the regions expected for two-nucleon and three-nucleon correlations, respectively.

to FSI effects.

Inclusive (e, $\left.\mathrm{e}^{\prime} \mathrm{pp}\right)$ and (e,e $\left.\mathrm{e}^{\prime} \mathrm{pn}\right)$ measurements on ${ }^{12} \mathrm{C}$ were carried out by Subedi et al. [23] using the Hall A set up at Jefferson Lab. The experiments used two high resolution spectrometers to measure the scattered electron and a high-energy emitted proton. The second correlated proton, or neutron, was detected in the large acceptance "Big Bite" spectrometer, or in a neutron detector array placed directly behind "Big Bite". These measurements were made at $\mathrm{Q}^{2}=2.0(\mathrm{GeV} / \mathrm{c})^{2}$ and $\mathrm{x}_{B}=1.2$. From the ratios of the measured cross sections it was deduced that $90 \%$ of correlated pairs in ${ }^{12} \mathrm{C}$ were proton-neutron pairs, $5 \%$ were proton-proton pairs and $5 \%$ were neutron-neutron pairs.

Further inclusive (e,e'pp) and semi-inclusive (e,e'p) measurements in ${ }^{12} \mathrm{C},{ }^{27} \mathrm{Al},{ }^{56} \mathrm{Fe}$ and ${ }^{208} \mathrm{~Pb}$ at $\mathrm{Q}^{2}>1.5(\mathrm{GeV} / \mathrm{c})^{2}$ and $\mathrm{x}_{B}>1.2$ by Hen et al. [24] were made with the Jefferson Lab CLAS detector. These measurements were anlaysed to extract the ratio of neutron-proton SRC pairs to proton-proton SRC pairs. It was found for all nuclei, irrespective of their neutronproton ratio, that proton-neutron pairs dominate by a factor of $\sim 20$. The dominance of protonneutron SRC has been attributed to tensor terms in the nucleon-nucleon interaction which favour a spin 1 , isospin 0 interaction.

Hen et al. noted that this has an interesting consequence for the high momentum fraction of protons and neutrons in heavy nuclei. Due to the substantial neutron excess in heavy nuclei, on average each proton will experience more SRCs than each neutron. This results in the protons having a larger high momentum fraction on average than neutrons.

Recent work by Duer et al. [25] has directly measured (e,e'pn) and (e,e'pp) reactions on the same nuclei as the previous study by Hen et al. [24], confirming the previous results and tying down the ratio of proton-proton SRC to neutron-proton SRC more precisely to $\sim 3 \%$.

It has long been known that the nuclear environment has an influence on the internal quark-gluon structure of nucleons [38]. Deep Inelastic Scattering (DIS) of leptons at high momentum transfer probes the internal quark structure of nucleons. The ratio of DIS cross sections in light, medium and heavy nuclei, compared to deuterium, is observed to decrease 
with increasing $\mathrm{x}_{B}$ over the range from $0.25<\mathrm{x}_{B}<0.75$. This phenomenon, known as the EMC effect, has been confirmed in both electron and muon scattering experiments. Recent measurements by Smookler et al. [26] have shown that the slope of the reduction in the DIS cross section ratio with $\mathrm{x}_{B}$ varies by a factor of two from -0.28 in ${ }^{12} \mathrm{C}$ to -0.54 in ${ }^{208} \mathrm{~Pb}$ [26]. This is interpreted as evidence that the external nuclear environment affects the momentum distribution of quarks inside individual nucleons.

Smookler et al. carried out this work in order to investigate whether there could be a connection between SRC and the EMC effect; i.e. could SRC affect the internal structure of nucleons in a way that explains the EMC effect. In this study DIS and quasi-elastic cross sections were measured simultaneously at the CLAS facility at Jefferson Lab for ${ }^{27} \mathrm{Al}$ and ${ }^{208} \mathrm{~Pb}$ and the data were analysed over a range of $\mathrm{x}_{B}$ for $\mathrm{Q}^{2}>1.5(\mathrm{GeV} / \mathrm{c})^{2}$ and $\mathrm{W}>1.8 \mathrm{GeV} / \mathrm{c}^{2}$.

Equating the DIS cross section to the structure function $F_{2}\left(x_{B}, Q^{2}\right)$, Smookler et al. split $F_{2}^{A}$ into terms corresponding to scattering off uncorrelated protons, uncorrelated neutrons and correlated SRC pairs [26]:

$$
F_{2}^{A}=\left(Z-n_{S R C}^{A}\right) F_{2}^{p}+\left(N-n_{S R C}^{A}\right) F_{2}^{n}+n_{S R C}^{A}\left(F_{2}^{p *}+F_{2}^{n *}\right),
$$

where $Z$ is the atomic number of the target, $N$ is the number of target neutrons and $n_{S R C}^{A}$ is the number of corrlated proton-neutron pairs measured in the target nucleus at the same values of $\mathrm{Q}^{2}$ and $\mathrm{x}_{B} . F_{2}^{p}$ is the structure function for free protons, $F_{2}^{n}$ is the structure function for free neutrons and $F_{2}^{p *(n *)}$ is an average modified structure function for protons (neutrons) in SRC pairs. Further, denoting the differences between the SRC-modified and free structure functions for the proton and neutron as $\Delta F_{2}^{p}=F_{2}^{p *}-F_{2}^{p}$ and $\Delta F_{2}^{n}=F_{2}^{n *}-F_{2}^{n}$, Eq. 1 becomes:

$$
F_{2}^{A}=Z F_{2}^{p}+N F_{2}^{n}+n_{S R C}^{A}\left(\Delta F_{2}^{p}+\Delta F_{2}^{n}\right) .
$$

Using measured DIS cross sections from deuterium and from heavier nuclei, together with the ratio of the SRC probabilities in the target nucleus compared to deuterium (deduced from simultaneously measured quasi-elastic cross section data), the quantity $n_{S R C}^{d}\left(\Delta F_{2}^{p}+\Delta F_{2}^{n}\right) / F_{2}^{d}$ can be extracted for each nucleus. This observable is shown in Fig. 11 as a function of $x_{B}$ for a range of heavy nuclei, including the measured ${ }^{27} \mathrm{Al}$ and ${ }^{208} \mathrm{~Pb}$ targets.

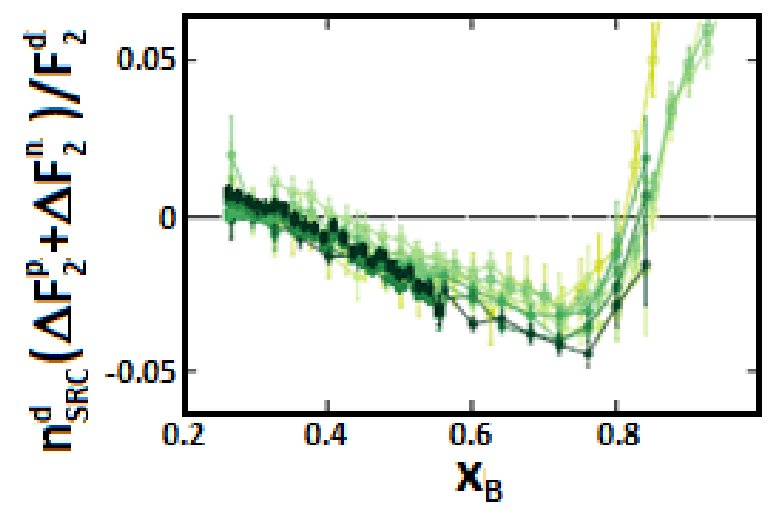

Figure 11: The modified ratio $n_{S R C}^{d}\left(\Delta F_{2}^{p}+\Delta F_{2}^{n}\right) / F_{2}^{d}$ (discussed in the text), plotted as a function of $\mathrm{x}_{B}$ for a range of light, medium and heavy nuclei, including ${ }^{27} \mathrm{Al}$ and ${ }^{208} \mathrm{~Pb}$ targets [26].

It is seen that the modified ratio $n_{S R C}^{d}\left(\Delta F_{2}^{p}+\Delta F_{2}^{n}\right) / F_{2}^{d}$ has a uniform magnitude and slope, independent of the host nucleus, over the range $0.25<\mathrm{x}_{B}<0.75$. The universality of this scaling suggests a strong connection between SRC and the EMC effect in nuclei, independent of the nucleus under study. 


\section{Photon-Induced 3-Nucleon Knockout Experiments}

In order to look at the role of photon absorption on three-nucleon clusters in more detail studies of three-nucleon knockout reactions were carried out.

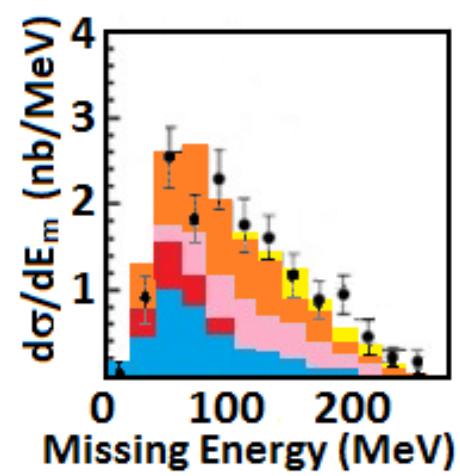

Figure 12: Missing Energy spectrum for the ${ }^{12} \mathrm{C}(\gamma, \mathrm{ppn})$ reaction for $\mathrm{E}_{\gamma}=300-400$ $\mathrm{MeV}$ [27], compared to calculations using the Valencia Model [31]. Light blue is photon absorption on two nucleons, with FSI; red indicates photon absorption on three nucleons, pink is photon absorption on three nucleons with FSI; orange represents quasi-free pion production where the pion is subsequently reabsorbed (as the $\mathrm{VM}$ is known to overestimate the strength of this process the orange region has been rescaled by a factor of 0.3 ). The yellow corresponds to quasi-free pion production where the pion rescatters.

Watts et al. [27] used the PiP/TOF experimental facility at Mainz to study the ${ }^{12} \mathrm{C}(\gamma, \mathrm{ppn})$ reaction, with one proton detected in the PiP trigger detector and two other nucleons detected in separate TOF detectors. Fig. 12 shows the 3-nucleon missing energy $\mathrm{E}_{3 m}$ spectrum obtained, compared with predictions of the VM. The VM does a good job of reproducing the strength and shape of the $E_{3 m}$ spectrum, after a correction has been applied to the strength of the pion production and reabsorption process. It is observed that while photon absorption on three nucleons is strongest at values of $\mathrm{E}_{3 m}<100 \mathrm{MeV}$, and FSI extend the three-nucleon absorption strength to higher values, together these two processes still constitute a small part of the overall cross section.

Watts et al. showed that further cuts on the invariant mass of two of the emitted nucleons could be used to suppress reactions involving pion production and reabsorption. These cuts were able to enhance the relative contribution from photon absorption on three-nucleon triples. However, these cuts drastically reduced the statistical accuracy of the remaining data sample and the three-nucleon contribution was less than half, even under the most favourable conditions.

Similarly Harty et al. studied the ${ }^{12} \mathrm{C}(\gamma, \mathrm{ppp})$ reaction [28] at photon energies up to 600 $\mathrm{MeV}$. While a three-nucleon absoption process could also be identified in this reaction, its contribution remained small compared to pion production plus FSI processes.

McAllister et al. [29], and Watts et al. [30], studied the ${ }^{12} \mathrm{C}(\gamma, \mathrm{pd})$ reaction with unpolarised and polarised photons, respectively, and compared their measurements to the ${ }^{3} \mathrm{He}(\gamma$,pd) twobody breakup reaction. Above $\mathrm{E}_{\gamma}=300 \mathrm{MeV}$, both reactions have a similar cross section dependnece on photon energy, with no indication of any influence from the $\Delta$ resonance. There are further similarities between the two reacions in that the photon asymmetry $\Sigma$ is positive and of similar values for photon energies between 200 and $260 \mathrm{MeV}$, although $\Sigma$ for the ${ }^{12} \mathrm{C}(\gamma$,pd) reaction diverges and becomes negative at higher photon energies. The recoil momentum distributions from the ${ }^{12} \mathrm{C}(\gamma, \mathrm{pd})$ reaction were compared with a simple model obtained by folding 
together 3 Elton-Swift wavefunctions in a relative S-state. These gave a good agreement with the experimental data, consistent with photon absorption on three nucleons.

\section{Summary and Conclusions}

The work reviewed in this paper has demonstrated that electromagnetically induced 2- and 3-nucleon knockout reactions from nuclei have contributions from several distinct processes. These include direct absorption on pairs of nucleons, absorption on nucleon pairs followed by final state interactions, direct absorption on 3-nucleon triples and absorption on 3-nucleon triples with final state interactions. Final state interactions can occur either with another nucleon in the pair or triple, or with other nucleons in the target nucleus. Pion production processes on a nucleon can also contribute to 2-nucleon and 3-nucleon emission. In this case the initial pion scatters, or is reabsorbed on a pair of nucleons, causing two or more nucleons to be emitted from the nucleus.

Exclusive 2-nucleon emission reactions may be selected by cuts on low missing energies $\mathrm{E}_{m}$. Taking into account typical reaction separation energies and experimental resolution, a cut of $\mathrm{E}_{m}<40 \mathrm{MeV}$ is able to select direct absorption on a pair of nucleons in valence orbitals in $1 \mathrm{p}$-shell nuclei, with the rest of the nucleus acting purely as a spectator. This is true for both $(\gamma, \mathrm{pn})$ and $(\gamma, \mathrm{pp})$ reactions. In the former case, the direct and exclusive 2-nucleon absorption process is indicated by a sharp peak in the missing energy spectrum. In the latter case, a strong signal is observed at low missing energies in the photon asymmetry spectrum.

Emission of one or more nucleons from more tightly bound 1s-shell orbitals, interactions involving more than two nucleons, final state interactions of the struck nucleons with other nucleons in the nucleus, pion emission mechanisms with rescattering or reabsorption, all shift strength to higher missing energies.

Strong differences are observed in the shape and the strength of the missing energy spectra, photon energy dependence, angular distributions and photon asymmetry measurements between $(\gamma, \mathrm{pn})$ and $(\gamma, \mathrm{pp})$ reactions. The largest difference is in the strength of the reaction where the $(\gamma, \mathrm{pp})$ reaction is around a factor of 30 weaker than the $(\gamma, \mathrm{pn})$ reaction. This is thought to be due to the suppression of neutral pion exchange currents in the $(\gamma, \mathrm{pp})$ reaction at low photon energies and the absence of tensor interactions, due to isospin symmetries, at higher energies.

Missing momentum spectra are found to be described well by a simple folding together of two single nucleon momentum spectra.

The direct processes, observed at low missing energies, include meson exchange currents and $\Delta$-currents as well as short range nucelon-nucleon correlations and final state interactions between the two participating nucleons. Experimentally it is not possible to separate out these distinct contributions. Rather, kinematic selections can be made which suppress certain of these mechanisms, providing a data sample which emphasises one, or other, mechanism. The strength of each component can only be deduced by comparison with theoretical models, which include all of the contributing processes.

As the contributing mechanisms are all strongly dependent on kinematic variables, the theoretical predictions have to be filtered through the experimental acceptances before a valid comparison with the measured data can be made. The theoretical predictions available are able to reproduce some of the energy, angular and isospin dependences seen in the data, but are not sufficiently good enough to allow the detailed properties of SRC to be extracted.

A fresh approach to the study of SRC was made by inclusive (e, $\left.\mathrm{e}^{\prime}\right)$ experiments at high $\mathrm{Q}^{2}$ and high $\mathrm{x}_{B}$. Under these conditions large amounts of energy and momentum are transferred to the target nucleus. The large quantities of energy transfer ensure that long-range processes 
such as $\Delta$-currents are suppressed, while large values of $\mathrm{x}_{B}$ ensure that the virtual photon is absorbed on particles with high momenta. As $x_{B}$ is increased it is expected that virtual photons will be absorbed on single nucleons, then correlated $2 \mathrm{~N}$ pairs and finally $3 \mathrm{~N}$ triples at the highest values. As the electron scattering cross sections decrease rapidly with increasing $\mathrm{x}_{B}$, the experimental data are presented as ratios of cross sections in heavy nuclei to cross sections in light nuclei. The data display similar behaviour in nuclei over a wide mass range. The plateaux observed in the ratios for $1<\mathrm{x}_{B}<2$ and $2<\mathrm{x}_{B}<3$ provide strong evidence of both $2 \mathrm{~N}$ SRC and $3 \mathrm{~N}$ SRC. Probabilities of interactions with $2 \mathrm{~N}$ pairs and $3 \mathrm{~N}$ triples have been extracted from the data.

Confirmation of virtual photon interactions with 2N SRC pairs was obtained in exclusive (e, $\left.\mathrm{e}^{\prime} \mathrm{pn}\right)$ and (e,e' $\mathrm{pp}$ ) experiments in which the observation of one high momentum nucleon was observed to be correlated with a second high momentum nucleon emitted in the opposite center-of-mass direction.

Further studies of the isospin dependence of $\left(\mathrm{e}, \mathrm{e}^{\prime} \mathrm{pN}\right)$ reactions showed that the neutron excess in heavy nuclei, coupled with dominance of interactions with proton-neutron pairs, compared to interactions with pairs of like nucleons, results in the protons having a larger high-momentum fraction than the neutrons.

More recent work has also suggested a connection between SRC and the EMC effect, in which the reduction in the DIS cross section, compared to deuterium, at large $\mathrm{x}_{B}$, is observed to be stronger in more massive nuclei. Separating out the DIS interaction with correlated nucleon pairs, reveals a universal dependence on $\mathrm{x}_{B}$, independent of the mass of the target nucleus.

$3 \mathrm{~N}$ interactions tend to occur at somewhat shorter ranges than $2 \mathrm{~N}$ interactions due to the involvement of a third nucleon. Both $3 \mathrm{~N}$ and $3 \mathrm{~N}+\mathrm{FSI}$ interactions contribute to $(\gamma, \mathrm{pn})$ and $(\gamma, \mathrm{pp})$ reactions at high $\mathrm{E}_{m}$. Comparisons of experimental missing energy spectra with the Valencia Model showed that variation of reaction kinematics could be used to enhance the $3 \mathrm{~N}$ contribution, but the $3 \mathrm{~N}$ contribution remained spread out over a range of missing energies and it also remained a relatively small fraction of the reaction cross section.

Photo-emission ( $\gamma, \mathrm{ppn})$ and ( $\gamma, \mathrm{ppp})$ reactions in which three nucleons were ejected from ${ }^{12} \mathrm{C}$ were also studied. The $3 \mathrm{~N}$ contribution to these reactions was found to occur at low $\mathrm{E}_{3 \mathrm{~m}}$ missing energies. It was shown that kinematic selections could be used to enhance the relative $3 \mathrm{~N}$ contribution in each reaction. However, $3 \mathrm{~N}$ and $3 \mathrm{~N}+\mathrm{FSI}$ processes generally remained a small part of the cross section. As in the case of $2 \mathrm{~N}$-emission reactions, estimating the strength of the $3 \mathrm{~N}$ contribution would require detailed comparison with theoretical models, filtered by the detector acceptance. Such comparisons have not so far been made.

\section{Acknowledgements}

The work reported in this paper is the result of efforts made by many colleagues, past and present, over a large number of years. I would particularly like to acknowledge the help and contributions provided by those physicists working in the A2 and A1 collaborations at Mainz and in the CLAS, CLAS12 and Hall A collaborations at Jefferson Lab, and by theorists from Valencia, Gent and Pavia.

Funding information The work reported in this paper has been supported by the UK Science and Technology Facilities Council through grants STFC 57071/1 and 50727/1. 


\section{References}

[1] L. Lapikás, Quasi-elastic electron scattering off nuclei, Nucl. Phys. A 553, 297 (1993), doi:10.1016/0375-9474(93)90630-G.

[2] J. D. Kellie, I. Anthony, S. J. Hall, I. J. D. MacGregor, A. McPherson, P. J. Thorley, S. L. Wan and F. Zettl, A tagged photon spectrometer for use with the mainz $180 \mathrm{MeV}$ microtron, Nucl. Instr. Meth. Phys. Res. A: Accel. Spectrom. Detect. Assoc. Equip. 241, 153 (1985), doi:10.1016/0168-9002(85)90526-1.

[3] I. Anthony, J. D. Kellie, S. J. Hall, G. J. Miller and J. Ahrens, Design of a tagged photon spectrometer for use with the Mainz $840 \mathrm{MeV}$ microtron, Nucl. Instr. Meth. Phys. Res. A: Accel. Spectrom. Detect. Assoc. Equip. 301, 230 (1991), doi:10.1016/0168-9002(91)90464-2.

[4] D. G. Middleton et al., ${ }^{3} \mathrm{He}\left(e, e^{\prime} p p\right)$ and ${ }^{3} \mathrm{He}\left(e, e^{\prime} p n\right)$ reactions at AmPS and MAMI, FewBody Syst. 44, 171 (2008), doi:10.1007/s00601-008-0283-x.

[5] D. G. Middleton et al., Investigation of the exclusive ${ }^{3} H e\left(e, e^{\prime} p n\right)^{1} H$ reaction, Phys. Rev. Lett. 103, 152501 (2009), doi:10.1103/PhysRevLett.103.152501.

[6] S. M. Doran et al., The ${ }^{4} \mathrm{He}(\gamma, 2 \mathrm{~N})$ reaction measured with tagged photons, Nucl. Phys. A 559, 347 (1993), doi:10.1016/0375-9474(93)90158-T.

[7] P. Grabmayr et al., Excitation functions for the two-nucleon photoabsorption in ${ }^{6} \mathrm{Li}$, Phys. Lett. B 370, 17 (1996), doi:10.1016/0370-2693(95)01581-7.

[8] S. N. Dancer et al., Investigation of the ${ }^{12} C(\gamma, p n)$ reaction using tagged photons, Phys. Rev. Lett. 61, 1170 (1988), doi:10.1103/PhysRevLett.61.1170.

[9] J. C. McGeorge et al., $(\gamma, 2 N)$ reaction in ${ }^{12} C$, Phys. Rev. C 51, 1967 (1995), doi:10.1103/PhysRevC.51.1967.

[10] G. E. Cross et al., Proton photoproduction from ${ }^{12}$ C, Nucl. Phys. A 593, 463 (1995), doi:10.1016/0375-9474(95)00318-U.

[11] P. D. Harty et al., The contribution of $2 N$ photon absorption in ${ }^{12} C(\gamma, 2 N)$ reactions for $E_{\gamma}$ = 150-400 MeV, Phys. Lett. B 380, 247 (1996), doi:10.1016/0370-2693(96)00508-4.

[12] Th. Lamparter et al., On photonuclear reaction mechanisms at intermediate energies, Z. Phys. A: Hadrons Nucl. 355, 1 (1996), doi:10.1007/s002180050069.

[13] I. J. D. MacGregor et al., Mechanisms in the ${ }^{12} C(\gamma, p n)$ and $(\gamma, p p)$ reactions, Phys. Rev. Lett. 80, 245 (1998), doi:10.1103/PhysRevLett.80.245.

[14] T.-H. Yau et al., The angular distribution of the ${ }^{12} \mathrm{C}(\gamma, p n)$ reaction for $E_{\gamma}=120-150 \mathrm{MeV}$, Eur. Phys. J. A 1, 241 (1998), doi:10.1007/s100500050055.

[15] S. Franczuk et al., ${ }^{12} C(\vec{\gamma}, p n)$ photon asymmetry for $E_{\gamma}=180-340 \mathrm{MeV}$, Phys. Lett. B 450, 332 (1999), doi:10.1016/S0370-2693(99)00184-7.

[16] D. P. Watts et al., The ${ }^{12} C(\gamma, N N)$ reaction studied over a wide kinematic range, Phys. Rev. C 62, 014616 (2000), doi:10.1103/PhysRevC.62.014616.

[17] C. J. Powrie et al., Polarized photon measurements of the ${ }^{12} C(\vec{\gamma}, p p)$ and ${ }^{12} C(\vec{\gamma}, p n)$ reactions for $E_{\gamma}=160-350 \mathrm{MeV}$, Phys. Rev. C 64, 034602 (2001), doi:10.1103/PhysRevC.64.034602 
[18] J. Robinson et al., Measurements of the ${ }^{12} C(\vec{\gamma}, p p)$ photon asymmetries for $E_{\gamma}=200-450$ $\mathrm{MeV}$, Eur. Phys. J. A 49, 65 (2013), doi:10.1140/epja/i2013-13065-0.

[19] I. J. D. MacGregor et al., The ${ }^{16} O(\gamma, 2 N)$ reaction measured with tagged photons, Nucl. Phys. A 533, 269 (1991), doi:10.1016/0375-9474(91)90490-W.

[20] D. Middleton et al., First measurements of the ${ }^{16} O\left(e, e^{\prime} p n\right){ }^{14} N$ reaction, Eur. Phys. J. A 29, 261 (2006), doi:10.1140/epja/i2005-10314-9 [Erratum: Eur. Phys. J. A 30, 469 (2006) doi:10.1140/epja/i2006-10131-8].

[21] D. Middleton et al., Knockout of proton-neutron pairs from ${ }^{16} \mathrm{O}$ with electromagnetic probes, Eur. Phys. J. A 43, 137 (2010), doi:10.1140/epja/i2009-10902-7.

[22] K. S. Egiyan et al., Measurement of two- and three-nucleon short-range correlation probabilities in nuclei, Phys. Rev. Lett. 96, 082501 (2006), doi:10.1103/PhysRevLett.96.082501.

[23] R. Subedi et al., Probing cold dense nuclear matter, Science 320, 1476 (2008), doi:10.1126/science.1156675.

[24] O. Hen et al., Momentum sharing in imbalanced Fermi systems, Science 346, 614 (2014), doi:10.1126/science.1256785.

[25] M. Duer et al., Probing high-momentum protons and neutrons in neutron-rich nuclei, Nature 560, 617 (2018), doi:10.1038/s41586-018-0400-z.

[26] B. Smookler et al., Modified structure of protons and neutrons in correlated pairs, Nature 566, 354 (2019), doi:10.1038/s41586-019-0925-9.

[27] D. P. Watts et al., Three-nucleon mechanisms in photoreactions, Phys. Lett. B 553, 25 (2003), doi:10.1016/S0370-2693(02)03186-6.

[28] P. D. Harty et al., Investigation of multiparticle final states in ${ }^{12} C$ photoreactions, Phys. Rev. C 57, 123 (1998), doi:10.1103/PhysRevC.57.123.

[29] S. J. McAllister et al., The ( $\gamma, p d)$ reaction in ${ }^{12} C$, Phys. Rev. C 60044610 (1999), doi:10.1103/PhysRevC.60.044610.

[30] D. Watts et al., Dependence of the ${ }^{12} C(\vec{\gamma}, p d)$ reaction on photon linear polarisation, Phys. Lett. B 64788 (2007), doi:10.1016/j.physletb.2007.02.013.

[31] R. C. Carrasco et al., Inclusive $(\gamma, N),(\gamma, N N)$ and $(\gamma, N \pi)$ reactions in nuclei at intermediate energies, Nucl. Phys. A 570, 701 (1994), doi:10.1016/0375-9474(94)90080-9.

[32] K. Livingston, The Stonehenge technique. A method for aligning coherent Bremsstrahlung radiators, Nucl. Instr. Meth. Phys. Res. A: Accel. Spectrom. Detect. Assoc. Equip. 603, 205 (2009), doi:10.1016/j.nima.2009.02.010.

[33] I. J. D. MacGregor et al., PiP - a large solid angle scintillation telescope for detecting protons and pions, Nucl. Instr. Meth. Phys. Res. A: Accel. Spectrom. Detect. Assoc. Equip. 382, 479 (1996), doi:10.1016/S0168-9002(96)00844-3.

[34] P. Grabmayr et al., The neutron Time-of-Flight detector system for $(\gamma, 2 N)$-studies at MAMIB, In Proc. Workshop on Future Detectors for Photonuclear Experiments, 225 (1991).

[35] G. Blanpied et al., New measurements of ${ }^{2} H(\vec{\gamma}, p) n$ and spin problems in coupled $N \Delta / N N$ interactions, Phys. Rev. C 52 R455 (1995), doi:10.1103/PhysRevC.52.R455. 
[36] S. Wartenberg, Die Strahlasymmetrie in der Deuteron-Photospaltung im Bereich von 160 bis $410 \mathrm{MeV}$, Ph.D. Thesis, University of Mainz (1998).

[37] J. Ryckebusch, M. Vanderhaeghen, L. Machenil and M. Waroquier, Effects of the finalstate interaction in $(\gamma, p n)$ and $(\gamma, p p)$ processes, Nucl. Phys. A 568, 828 (1994), doi:10.1016/0375-9474(94)90362-X.

[38] J. J. Aubert et al., The ratio of the nucleon structure functions $F_{2}^{N}$ for iron and deuterium, Phys. Lett. B 123, 275 (1983), doi:10.1016/0370-2693(83)90437-9. 